\title{
Assessing and Enhancing Standards Education for Environmental Manage- ment and Sustainability
}

\section{Dr. Deanna H. Matthews, Carnegie Mellon University}

Dr. Deanna H. Matthews is Associate Department Head for Undergraduate Affairs and Associate Teaching Professor in Engineering and Public Policy at Carnegie Mellon University. She serves as the academic advisor to undergraduate students in the department and teaches introductory and capstone courses for engineering students to understand the complex nature of technology solutions in society. Her research interests include developing student meta-cognition and assessment of individual student work in projectbased assignments. She received her B.S.E. in Civil Engineering from Duke University (1994) and her M.S. (1995) and Ph.D. (2001) in Civil and Environmental Engineering from Carnegie Mellon University.

\section{Prof. H. Scott Matthews, Carnegie Mellon University}

H. Scott Matthews is a Professor in the Departments of Civil and Environmental Engineering and Engineering \& Public Policy and the Research Director of the Green Design Institute at Carnegie Mellon University. The Green Design Institute is an interdisciplinary research consortium at Carnegie Mellon focused on identifying and assessing the environmental impacts of systems and helping businesses manage their use of resources and toxic materials.

His research and consulting interests are in the area of valuing the socio-economic implications of environmental systems and infrastructure and industrial ecology. Of particular interest are using the Internet to facilitate environmental life cycle assessment of products and processes, estimating and tracking carbon emissions across the supply chain, and the sustainability of infrastructure. At Carnegie Mellon, he has taught graduate and undergraduate courses in the Departments of Economics, Civil and Environmental Engineering, Engineering and Public Policy, and Computer Science. 
Incorporating Standards Education into Courses on Environmental Management and Sustainability

Abstract: In this work, we discuss the process and outcome of incorporating standards education into courses on environmental management and sustainability. The research involved a pre-assessment of student knowledge about the ISO 14040 Life cycle Assessment standards, examining the coverage of standards materials in courses, and assessing gains in standards knowledge through a post-assessment. Participants included students from several universities where courses in LCA, environmental management and sustainability are taught annually. Each course uses the same on-line textbook to teach various concepts associated with LCA. Over the period of research, we expanded and enhanced the content related to engineering standards, the standards making process and organizations, the ISO 14040 LCA standards, and its uses in professional practice. Comparisons are made in individual student gains pre- and post-course, and in cohort gains prior to changing the content in the textbook.

This work helped to fill a gap in teaching environmental performance assessment by formalizing curricular elements associate with the development of the underlying standards, as well as how those standards enable the comparative assessments of processes, products, and companies. This will allow many people, beginning at the undergraduate level, to understand the important role that standards have played, and continue to play, in this domain. They will also see how the critical interconnected development of standards and practice are enabling innovative sustainable opportunities to understand and improve environmental performance.

Introduction

The ISO 14040 standard for Life Cycle Assessment is a foundational standard for practitioners in the area of environmental benchmarking and comparative assessment. Students studying environmental management and sustainable engineering need to be aware of this standard and have a more in-depth understanding of its definitions and requirements as they move to the workplace. Our work developed materials on ISO 14040 standards to be used in course materials, with an investigation of how including these materials might impact student learning on the topic. This paper demonstrates our method for enhancing standards education through materials development. We first discuss the development of standards for benchmarking and comparative assessment, and various courses and curricula that include these materials. Next we describe our process for assessing student knowledge of standards and developing standards based material. We end with some lessons learned about the process for developing materials on standards.

Standards for Environmental Performance

There is increasing global demand for benchmarking and comparative assessment of the environmental performance of companies including that of specific products and 
processes. This information is becoming more important to consumers and decisionmakers in industry, society, and government worldwide. These performance measurements have been achieved through development of metrics and methods to consider use of energy and other material and resource inputs, as well as outputs such as air and water emissions and releases like wastes. A relatively recent component of this performance assessment has included consideration of sustainability, which is often broadly defined as the ability of current generations to meet their needs without compromising the ability of future generations to meet their needs [1]. Sustainability has more colloquially been defined as the interaction of economic, environmental, and social factors. Companies have thus been more broadly seeking ways to report their performance across this holistic view of their operations and led to outcomes like annual sustainability reports for major corporations around the world. For companies operating in certain jurisdictions, reporting of this kind is mandatory, but in most cases is done on a voluntary basis as a means of driving shareholder value by creating a perception that the company is proactively measuring their performance and developing competitive advantage.

At present, companies are largely focused on reporting the energy consumption, water use, and greenhouse gas (GHG) emissions associated with their company overall, as well as on a product or process level. The US EPA's GHG Reporting Program (GHGRP) launched mandatory reporting at the facility level for many energy-intensive sectors, which has caused approximately 10,000 facilities per year to quantify their carbon emissions. In support of such efforts, global bodies have defined methods to quantify the energy, environmental, and other impacts at various levels of resolution. Companies are reporting their performance at unprecedented levels to agencies using mandatory and voluntary data systems.

At the company level, documentary standards have been created by groups like the World Resources Institute (WRI) to define how firms should measure their overall "carbon footprint" of GHG emissions from their own in-company activities (called scope 1), that of their energy suppliers (scope 2), and for all other upstream and downstream activities (scope 3) [2], [3]. Once the emissions have been quantified, firms report resulting GHG emissions through voluntary or mandatory data collection efforts like the Carbon Disclosure Project or EPA's GHGRP. Building on the foundation of these standards, WRI has also developed standards for reporting GHG emissions at the product-specific level [4]. Finally, ISO has developed standards specific to carbon and water footprinting (ISO 14067, ISO 14046).

The aforementioned standards fundamentally depend on the definitions and comprehensive environmental analysis contained in the ISO 14040 standards for life cycle assessment (LCA). These standards, first formalized in the 1990s, provide a systematic quantified inventory and impact assessment of the environmental implications of products in every life-cycle stage: materials extraction, manufacturing, use, and 
ultimate fate of the product (reuse, recycling, incineration, or landfilling). Based on the environmental assessment, improvements could be made to products or processes to minimize their environmental footprint over their life cycle.

A number of groups worldwide have developed LCA approaches and databases. In the US, the Society for Environmental Toxicology and Chemistry (SETAC) and the Environmental Protection Agency (EPA) spearheaded the development efforts. Most current LCA methods are based on bottom-up process models that identify and quantify resource inputs and environmental outputs at each life cycle stage based on mass-balance calculations. This approach requires collection of detailed data directly from companies or studies published in literature. Other LCA methods include holistic top-down studies of an entire economy using input-output tables and emissions factors from publicly available data. As a checkpoint, a web-based input-output LCA tool that we created in 2000 has been used about 3 million times (www.eiolca.net).

Since the LCA Standard has created all of the underlying definitions and terminology to be used to assess environmental performance for specified functional units, it was the natural building block for all of the other standards mentioned above. Thus, the ISO 14040 LCA standard should be a focus of instruction for practitioners and students in this field.

\section{U.S. Sustainability Courses and Curricula}

Despite the large investments in the underlying standards, as well as supporting methods to implement them and to create robust decision tools, most are unaware of the critical role of standards and the standardization of methods needed to support all of these process, product, and firm-level performance measurements. Results of national sustainable engineering curriculum assessments in which our team collaborated showed that five years ago there were only a few whole semester undergraduate or graduate courses on life cycle assessment (LCA) in 100 engineering colleges in the US. Most science and engineering programs that discussed or taught LCA did so in 2-3 lecture course modules focused on LCA data collection and organization and simple modeling exercises [5], [6]. An identified gap was that there were no centralized teaching resources such as textbooks or lecture materials available in this domain.

Based on this outcome, members of our team led the development over the past several years of a free online textbook [7] (available at lcatextbook.com) to teach various concepts associated with LCA to undergraduate and graduate students. It has been developed to support entire semester LCA courses, as well as the short course modules historically used, and short course or executive education formats. Chapter resources include learning objectives and homework questions, as well as tailored interactive spreadsheet and web models. For instructors, we have developed PowerPoint lecture note files, and are developing video lectures (approximately 1 hour total per session). We developed and disseminated all of these materials in electronic and web-based formats to 
ensure a long-term and low-cost sustainable delivery platform. When we refer to 'the textbook', we refer to not just the text itself, but also this entire ecosystem of learning resources.

As a result of these investments, the book has already been adopted in around 50 undergraduate or graduate full-semester courses at universities in the US, leading to thousands of downloads of the textbook, with adoptions increasing every semester. For example, the Spring 2018 semester had 1,000 full book downloads. These courses are in science, engineering, social science, and management departments. The online textbook and website is being used on 4 continents, and being translated into three languages. In short we have already curated an international network of professors and professionals using the LCA textbook as well as the supplemental learning resources that form a natural audience for further educational material.

ISO 14040 Standards Content

The textbook included from the beginning material on ISO 14040. However, we recognized the need for further developing this content to make it more robust and useful for future practitioners. Better understanding of both the standards development process and the standards text and uses would need to be included. For example, while the details of the ISO LCA Standard are a central component, the development of the specific Standard as well as the general way in which Standards are created, or in the case of greenhouse gas protocols the interaction of multiple standards, was not a part of curricula or course content.

Through our project, we further developed the standards content and fill a significant gap in the teaching of environmental performance assessment (such as LCA) by formalizing curricular elements associated with the development of the underlying standards, as well as how those standards enable the comparative assessments of processes, products, and companies. This will allow many people, beginning at the undergraduate level, to understand the important role that standards have played in this domain. They will also see how the critical interconnected development of standards and practice are enabling innovative sustainable opportunities to understand and improve environmental performance. Specifically, based on our unique experience in assessing curricula needs and developing teaching resources related to LCA, we identified the following four aspects which were integrated into newly developed materials:

1) The historical drivers for standardization in the environmental performance domain, the organization of the various entities (ISO, ASTM, etc.) that interact to produce standards, and the role of standards in increasing competitiveness and spurring sustainable innovation of products, processes, and firms.

2) The difference between documentary and measurement standards. In the environmental performance domain, they are generally accounting rather than measurement standards. While the overall carbon footprint of a company may be 
comprised of many individual components that can be measured in some way (e.g., tailpipe or stack measurements of emissions), the overall combination is done through accounting methods.

3) The 'map' between the standard and the study document. Current courses mention the standards, their development, and use, but do not directly integrate use of the standard in the course. This presents the Standard as secondary to the methods used, even though following the standards is essential for an ISO-compliant LCA or carbon footprint report. Since many students will go on to be practitioners by completing LCAs based on the standards, or reviewing LCAs according to the standards, it is important for them to recognize the complexity and rigor these standards have.

4) Understanding the role of professional peer review for standard compliance. ISO LCA studies must be peer reviewed by external parties if they will support comparative assessments intended for the public (which many are). Learning how peer review is done, and the role of critical feedback, is a critical piece of the standard process. This means both writing peer reviews and reading/ comprehending existing reviews.

\section{Study Method}

The research involved study over a 2 year period. We asked faculty who taught a full-semester life cycle assessment course that used our textbook as a resource to participate and eleven faculty at different institutions volunteered. These institutions included eight doctoral, highest research level universities, and one each of doctoral - higher research, doctoral - moderate research, and masters institutions. The courses included mostly courses for graduate students but included undergraduate enrollment. Students from eight institutions completed assessments.

In year one, we collected pre- and post-assessment survey data from students in courses at these institutions. Year one data provides a baseline of knowledge in standards content for life cycle assessment. At the end of year one, after discussion with LCA practitioners and standards colleagues, we made changes to the textbook to improve standards content. During year two, we repeated the pre- and post-assessment surveys for students in the courses. Thus we have pre- and post-assessments of a cohort of students before the "intervention" of textbook improvements, and pre- and post-assessments of a cohort of students after the "intervention" of textbook improvements.

The pre- and post-assessment included a set of questions to gauge student knowledge and awareness of both life cycle assessment generally (12 questions), and life cycle assessment standards specifically ( 24 questions, full assessment provided at end of the paper). The survey instrument was the same for both assessments, with the post-assessment also including questions about the chapters of the textbook that the student read and which ones were assigned. The latter questions help us to identify whether the standards content was emphasized in their particular course or not, at least according to the student. 
Students were solicited via an email forwarded from the study team to the course instructor who then forwarded it to their students either directly or through a course management system. In the surveys, students were asked for their name, email, and institution in order to match pre- and post-assessment results. We also used emails from the pre-assessments to directly email students the post-assessment link. A total of 135 students completed the pre-assessment, and 35 students completed the post-assessment, with 29 students completing both surveys. Students were not compensated for their time, and unless their instructor made other arrangements they were not rewarded or penalized in their course for completing either assessment. We did not share information about students completing the assessment with course instructors.

Results

On the pre-assessment, we find that students across all three semesters had similar prior knowledge about life cycle assessment topics and life cycle assessment standards issues. Mean scores were 21, 21, and 20 points (out of 36) for students from the three semesters, respectively. We divided the questions on the assessment into "general LCA knowledge" questions and "LCA standards knowledge" questions. No difference in the mean scores or standard deviations across the three semesters is found. Students answer $\sim 7$ of the 12 general questions correctly, and $\sim 13$ of the 24 standards questions correctly.

We next examined scores for all of the students responding to the post-test, not matching students across both assessments. Overall, we find that students did better on the post test generally - scores were 26, 22, and 26 (out of 36) for students from the three semesters respectively. The improvement in scores is due to increased correct answers on the LCA standards question set. Students gained $\sim 1$ point on the general questions, but 2-4 points on the standards questions. Correspondingly, when looking at the scores for an individual question, we see that students responded correctly more often on the post-assessment than on the preassessment. Table 1 shows the percentage of students answering each question correctly. Note that for some questions, students were given the option to "select all that apply" so multiple responses could have been recorded. We give an overall score for that question (100\% would mean a student selected only the correct boxes and none of the incorrect boxes), as well as the scores for the individual elements of a question (the percentage of students identifying one of the options as correct response, or not selecting an incorrect response). Few students were able to get all elements correct as reflected in the overall score, whereas certain elements were easily identified as the correct response.

Table 1. Pre- and Post-Assessment Scores

\begin{tabular}{|c|c|c|}
\hline Question & Pre-assessment percent correct & Post-assessment percent correct \\
\hline $\begin{array}{l}\text { Which of the following are examples } \\
\text { of life cycle thinking? }\end{array}$ & $73 \%$ & $83 \%$ \\
\hline $\begin{array}{l}\text { Which of the following are examples } \\
\text { of activities of initial costs to be } \\
\text { included in a life cycle cost model of } \\
\text { a building (you may choose more } \\
\text { than one)? }\end{array}$ & $\begin{array}{c}67 \% \\
(82 \%, 90 \%, 46 \%, 56 \%)\end{array}$ & $\begin{array}{c}73 \% \\
(86 \%, 89 \%, 33 \%, 50 \%)\end{array}$ \\
\hline
\end{tabular}




\begin{tabular}{|c|c|c|}
\hline $\begin{array}{l}\text { Which of the following are part of } \\
\text { the LCA standard (choose all that } \\
\text { apply) }\end{array}$ & $\begin{array}{c}77 \% \\
(86 \%, 73 \%, 95 \%, 55 \%)\end{array}$ & $\begin{array}{c}94 \% \\
(100 \%, 92 \%, 100 \%, 83 \%)\end{array}$ \\
\hline $\begin{array}{l}\text { Which of the following activities } \\
\text { were done to produce the LCA } \\
\text { Standard (choose all that apply)? }\end{array}$ & $\begin{array}{c}53 \% \\
(56 \%, 82 \%, 56 \%, 33 \%, 38 \%)\end{array}$ & $\begin{array}{c}65 \% \\
(78 \%, 94 \%, 56 \%, 50 \%, 47 \%)\end{array}$ \\
\hline $\begin{array}{l}\text { ISO } 14040 \text { is an example of which } \\
\text { type of standard? }\end{array}$ & $48 \%$ & $75 \%$ \\
\hline $\begin{array}{l}\text { Why is it important to follow a } \\
\text { standard when doing environmental } \\
\text { comparisons? }\end{array}$ & $88 \%$ & $100 \%$ \\
\hline $\begin{array}{l}\text { Which of the following activities are } \\
\text { required as part of the LCA } \\
\text { Standard? (choose all that apply) }\end{array}$ & $\begin{array}{c}55 \% \\
(56 \%, 87 \%, 24 \%, 30 \%, 76 \%)\end{array}$ & $\begin{array}{c}70 \% \\
(67 \%, 100 \%, 58 \%, 36 \%, 89 \%)\end{array}$ \\
\hline $\begin{array}{l}\text { Which of the following is not a study } \\
\text { design parameter in the LCA } \\
\text { Standard }\end{array}$ & $35 \%$ & $67 \%$ \\
\hline $\begin{array}{l}\text { In what order does the LCA Standard } \\
\text { suggests dealing with a hypothetical } \\
\text { multifunctional system involving a } \\
\text { refinery system. Select } 1 \text { for the first } \\
\text { method, } 2 \text { for the second method, and } \\
3 \text { for the third method. }\end{array}$ & $\begin{array}{c}14 \% \\
(24 \%, 30 \%, 14 \%)\end{array}$ & $\begin{array}{c}27 \% \\
(44 \%, 39 \%, 53 \%)\end{array}$ \\
\hline $\begin{array}{l}\text { Which of the following is true about } \\
\text { input-output models for LCA? (select } \\
\text { all that apply) }\end{array}$ & $\begin{array}{c}33 \% \\
(55 \%, 56 \%, 53 \%)\end{array}$ & $\begin{array}{c}37 \% \\
(67 \%, 58 \%, 61 \%)\end{array}$ \\
\hline $\begin{array}{l}\text { Why are process matrix models } \\
\text { useful in LCA? (Select all that apply) }\end{array}$ & $\begin{array}{c}47 \%, \\
(49 \%, 81 \%, 70 \%, 36 \%)\end{array}$ & $\begin{array}{c}58 \% \\
(72 \%, 86 \%, 86 \%, 44 \%)\end{array}$ \\
\hline $\begin{array}{l}\text { What is true about life cycle impact } \\
\text { assessment (LCIA) in the LCA } \\
\text { Standard? }\end{array}$ & $39 \%$ & $47 \%$ \\
\hline $\begin{array}{l}\text { How is peer review of an LCA } \\
\text { addressed in the ISO Standard? } \\
\text { (Select all that apply.) }\end{array}$ & $\begin{array}{c}40 \% \\
(42 \%, 73 \%, 87 \%)\end{array}$ & $\begin{array}{c}47 \% \\
(61 \%, 86 \%, 89 \%)\end{array}$ \\
\hline
\end{tabular}

When looking at scores for students with matched pre-assessment and post-assessment surveys (29 total), we can examine gains more closely. On average, these students increased their scores an average of 4 points or about a 10\% increase in score from the pre- to the post-assessment. The majority of those gains came on answering standards questions correctly on the post-assessment. Students in the final semester, who were using the text with additional and updated standards content, increased their scores on the standards based questions more than those in the prior semesters but the difference is not significant.

Student responses to the open ended question - "How would the ISO 14040 standard be useful for completing this LCA task from your boss, if at all?" indicated a gain in awareness of standards in the LCA area. On the pre-assessment, 51\% of the students responded "don't know" or "unsure" or left the question blank. On the post-assessment, no students answered don't know or unsure, but nine students left the question blank (25\%). On the pre-assessment, $30 \%$ of students had a response that generally referred to standards, and $13 \%$ had responses that 
indicated some knowledge of LCA. On the post-assessment, 33\% had a response that generally referred to standards, and 39\% had responses that indicated some knowledge of LCA. These responses included comments about the elements of an LCA that the standard would address, the fact that the standard would promote consistency, and identified the need for using the standard to allow comparisons across different products.

Conclusions, Impacts and Outcomes

While we cannot extract any statistically significant results, we do see an apparent gain in knowledge from the pre-assessments to the post-assessments. These gains could be due to differences in the students' work ethic and success in the individual courses, and selection bias of students who are generally doing well in the class being willing to respond to the postassessment request. We may also be seeing an indirect effect of awareness to the faculty member extended to the students. Since the faculty instructors were receiving our correspondence about the research project and changes to the textbook in the area of standards, we may be seeing the general effect of these instructors highlighting the standards material in their courses.

The materials added (detailed above) include content on an array of standards knowledge. We emphasize the market orientation of the standards, from both the development and use perspectives. We provide students with linkages from the standard text to the work in LCA that they would be performing or reviewing, thus giving them an integrated view of the role standards will likely have in their future positions in LCA. These areas are consistent with suggested standards content areas [8], [9]. While one chapter of the textbook is specifically focused on the ISO standard, we incorporate references to the standard in subsequent chapters to reinforce the need to be knowledgeable about this content. The students may also have used resources (provided by the faculty instructor or not) that include standards content.

One difference in this approach is the potential reach of the materials. Rather than creating a single module on standards, we created centralized materials in an existing textbook and course materials environment. Within the scope of our project, we had participation from 8 courses reaching approximately 300 - 500 students over the 3 semesters of courses with correspondence about the project that was standards based. We made improvements to the textbook chapter on standards and increased references to standards in other chapters. Outside the scope of the project, we are aware of at least twenty (20) different courses that are using our textbook, with an annual enrollment of around 2,000 - 3,000 students. Since we do an annual update of the text, and notify instructors on our mailing list annually of changes, we have made these instructors more aware of the need to cover standards content in their courses. So, through this model, we hope to have a far reaching impact on standards awareness among students and faculty alike.

Some lessons learned from the assessment project are to provide students several options to complete the post-assessment instrument. The low end-of-semester response rate was disappointing, albeit not surprising. We of course would have preferred a more extensive assessment tool rather than the brief instrument we created in order to get a more robust idea of what the students know at the end of the course. We see some of the questions with low correct 
response rates that would reflect coverage in course content - for example, few students are able to answer the question about dealing with a multifunctional system through system expansion. Since instructors may not be address system expansion in a course, since it is an advanced topic, the students may not be exposed to this content to be able to answer correctly.

The modest gains that students made reinforce the need for faculty to emphasize standards in the classroom setting. We cannot guarantee that students in these courses, or any courses that use the text, are reading the material to a depth of understanding to have the broad standards awareness that is needed. We hope that by including repeated references to the standard in different portions of the text we begin to address this lack of emphasis.

\section{Acknowledgements}

This work was supported by a Standards Services Curricula Development Cooperative Agreement \#70NANB15H338 awarded by the U.S. Commerce Department's National Institute of Standards and Technology (NIST).

References

[1] World Commission on Environment and Development, A Future for All (Brundtland Commission Report), London: Oxford Press, 1988

[2] World Resources Institute, "The Greenhouse Gas Protocol: A Corporate Accounting and Reporting Standard", 2001.

[3] World Resources Institute, "The Greenhouse Gas Protocol: Corporate Value Chain (Scope 3) Accounting and Reporting Standard", 2011.

[4] World Resources Institute, "The Greenhouse Gas Protocol: Product Life Cycle Accounting and Reporting Standard", 2011.

[5] C. Murphy, D. Allen, B. Allenby, J. Crittenden, C. Davidson, C. Hendrickson, H. S. Matthews, "Sustainability in Engineering Education and Research at U.S. Universities”, Environmental Science \& Technology, 2009, 43 (15), pp. 55585564, 2009.

[6] M. Bilec, C. Hendrickson, A. Landis and H. S. Matthews, 'Updating the Benchmark Sustainable Engineering Education Report - Trends from 2005 to 2010', Proceedings of the 2011 ASEE Conference, Vancouver, British Columbia, Canada, June 26-29, 2011, 7 pages.

[7] H. S. Matthews, C. Hendrickson, and D. Matthews, Life Cycle Assessment: Quantitative Approaches for Big Decisions, http://www.lcatextbook.com/, 2015. 
[8] C. Leachman and C. Pezeshki, "What's Standard? Industry Application versus University Education of Engineering Standards," Proceedings of the 2015 ASEE Conference, Seattle Washington, June 14-17, 2015, 13 pages.

[9] J. Olshefsky, "Standards Education: Bridging the Gap Between Classroom Learning and Real World Applications," Proceedings of the 2010 ASEE Conference, Louisville, Kentucky, June 20-23, 2010, 9 pages. 
1. Which of the following are examples of life cycle thinking?

Considering if battery powered vehicles can improve air quality compared to regular vehicles.

Producing a pencil

Burning gasoline in an engine

2. Which of the following are examples of activities of initial costs to be included in a life cycle cost model of a building (you may choose more than one)?

Design of the building

Construction of the building

Energy used by occupants of the building

Costs of financing

3. Which of the following are part of the LCA standard (choose all that apply)

Goal and Scope Definition

Inventory Analysis

Impact Assessment

Interpretation

4. Which of the following activities were done to produce the LCA Standard (choose all that apply)?

Text drafted

Consensus discussions

Voting on suggestions

Market analysis

Adoption by industry

5. ISO 14040 is an example of which type of standard?

Measurement standard

Documentary standard

Testing method 
6. Why is it important to follow a standard when doing environmental comparisons?

To reduce magnitude of impacts

Consistency and comparability across different studies

Ability to quantify carbon footprints

7. Which of the following activities are required as part of the LCA Standard? (choose all that apply)

Public release

Documentation of assumptions

Use of primary data

Use of a process flow diagram

Use of a hybrid LCA model

8. Which of the following is not a study design parameter in the LCA Standard

Goal of study

Functional Unit

Process Flow Diagram

Boundary

9. In what order does the LCA Standard suggests dealing with a hypothetical multifunctional system involving a refinery system. Select 1 for the first method, 2 for the second method, and 3 for the third method.

\begin{tabular}{|l}
\hline Allocate by economic value \\
Allocate by energy \\
$\square$ System expansion \\
\hline
\end{tabular}

10. Which of the following is true about input-output models for LCA? (select all that apply)

Direct results are those of the first tier of suppliers

Indirect results are larger than direct results

They are best used as a screening tool 
11. Why are process matrix models useful in LCA? (Select all that apply)

They rely on process-based data

They consider effects across whole system of processes

They are better than input-output models

They are available in software tools like SimaPro

12. What is true about life cycle impact assessment (LCIA) in the LCA Standard?

Use of at least one LCIA method is required in an LCA

Studies may not consider only global warming as an impact

Global warming impacts must be considered using IPCC methods

13. How is peer review of an LCA addressed in the ISO Standard? (Select all that apply.)

It is a required part of any study.

It is required when results are to be used for comparisons released to the public

Experts must be paid to review.

14. Your boss asks about doing an LCA on a new product and asks you to explain how to do one. Give a short overview (less than 100 words) of how you would complete the work and use it to compare against a competitor.

15. How would the ISO 14040 standard be useful for completing this LCA task from your boss, if at all?

16. Name one specific concept that you felt that was not explained well enough in the textbook or lecture that should be improved for next year. 
17. For each chapter, tell me when you read it. Please be honest.

\begin{tabular}{|c|c|c|c|c|}
\hline & Never Read & Only Skimmed & Read Before Lecture & $\begin{array}{c}\text { Read While Doing } \\
\text { Homework }\end{array}$ \\
\hline \multicolumn{5}{|l|}{ Chapter 1 (Intro) } \\
\hline \multicolumn{5}{|l|}{$\begin{array}{l}\text { Chapter } 2 \text { (Quantitative } \\
\text { Methods) }\end{array}$} \\
\hline \multicolumn{5}{|l|}{$\begin{array}{l}\text { Chapter } 3 \text { (Life Cycle } \\
\text { Cost) }\end{array}$} \\
\hline \multicolumn{5}{|l|}{$\begin{array}{l}\text { Chapter } 4 \text { (LCA } \\
\text { Standard) }\end{array}$} \\
\hline \multicolumn{5}{|l|}{$\begin{array}{l}\text { Chapter } 5 \text { (LCA Data } \\
\text { and Process Flow } \\
\text { Diagrams) }\end{array}$} \\
\hline \multicolumn{5}{|l|}{$\begin{array}{l}\text { Chapter } 6 \\
\text { (Allocation/System } \\
\text { Expansion) }\end{array}$} \\
\hline \multicolumn{5}{|l|}{$\begin{array}{l}\text { Chapter } 8 \text { (Input-Output } \\
\text { Models) }\end{array}$} \\
\hline \multicolumn{5}{|l|}{$\begin{array}{l}\text { Chapter } 9 \text { (Process } \\
\text { Matrix Models) }\end{array}$} \\
\hline \multicolumn{5}{|l|}{ Chapter 10 (LCIA) } \\
\hline \multicolumn{5}{|l|}{$\begin{array}{l}\text { Chapter } 11 \\
\text { (Uncertainty) }\end{array}$} \\
\hline $\begin{array}{l}\text { Chapter } 12 \text { (Structural } \\
\text { Path Analysis) }\end{array}$ & & & & \\
\hline
\end{tabular}


18. For each chapter, how helpful was the textbook content in explaining concepts and improving your knowledge about that LCA topic?

Chapter 1 (Intro)
Chapter 2 (Quantitative
Methods)
Chapter 3 (Life Cycle
Cost)
Chapter 4 (LCA
Standard)
Chapter 5 (LCA Data
and Process Flow
Diagrams)
Chapter 6
(Allocation/System
Expansion)
Chapter 8 (Input-Output
Models)
Chapter 9 (Process
Matrix Models)
Chapter 10 (LCIA)
Chapter 11
(Uncertainty)
Chapter 12 (Structural
Path Analysis)

19. Your Name. We will use this information to aid in matching your post-assessment responses with your pre-assessment responses only.

20. Your University/Institution. We will use this information to aid in matching your post-assessment responses with your pre-assessment responses only.

21. Email Address. We will use this information to aid in matching your post-assessment responses with your pre-assessment responses only. 